\title{
Effect of EDTA on Growth of 4-Dimethylamino- $N$-Methyl-4-Stilbazolium Tosylate Crystals by Slope Nucleation Method
}

\author{
Jer-I Wu, Rengasamy Gopalakrishnan, Clifford I-Der TaI and Chung-Wen LaN* \\ Department of Chemical Engineering, National Taiwan University, Taipei, Taiwan 10617, ROC \\ (Received November 27, 2003; accepted January 9, 2004; published April 9, 2004)
}

Nucleation experiments (metastable zone width) were carried out for 4-dimethylamino- $N$-methyl-4-stilbazolium tosylate (DAST) solutions at different saturation temperatures with and without the addition of a chelating agent. Using the results obtained from metastable zone width experiments, the slope nucleation method growth of DAST was performed. It was revealed that the addition of the chelating agent EDTA significantly suppresses spurious nucleation and cluster formation in the DAST solution, so that fewer, but larger, crystals can be easily obtained. With EDTA, the crystals grown also showed better crystallinity, hardness, and transparency in the visible/near-infrared region. [DOI: 10.1143/JJAP.43.1507]

KEYWORDS: nucleation, chelating agent, metastable zone, solubility, growth from solutions, single-crystal growth, DAST, organic salt, nonlinear optic materials

\section{Introduction}

Organic nonlinear optical (NLO) materials have attracted considerable attention and have been extensively studied owing to their higher nonlinear optical coefficients and faster electrooptic response than those of inorganic materials. ${ }^{1,2)}$ Many new organic crystals have been found based on predictive molecular engineering approaches and have been shown to have potential applications in nonlinear optics. In recent years, considerable research effort has been exerted in exploring novel organic materials. ${ }^{3-5)}$ The new organic NLO crystal 4-dimethylamino- $N$-methyl-4-stilbazolium tosylate (DAST) was discovered by Nakanishi et al. ${ }^{6,7)}$ and shows excellent properties. DAST belongs to the monoclinic crystal system with the non-centrosymmetric space group $\mathrm{C}_{\mathrm{c}}$ and the point group $\mathrm{m}$, and has the following lattice parameters: $a=10.365 \AA, b=11.322 \AA, \quad c=17.893 \AA$, $Z=4$ and $\beta=92.24^{\circ} .{ }^{8)}$ DAST also exhibits a large electrooptic coefficient $r_{111}=77 \pm 8 \mathrm{pm} / \mathrm{V}$ with a low dielectric constant, ${ }^{9,10)}$ and can be used in second-harmonic generation, ${ }^{8,11)}$ terahertz frequency generation, ${ }^{12)}$ telecommunication, ${ }^{13,14)}$ and other applications. ${ }^{2,15)}$

Although DAST crystals have shown excellent NLO properties in many applications, the growth of high-quality large crystals has been a challenging task. Pan et al. ${ }^{9)}$ and Sohma et al. ${ }^{15)}$ have illustrated the feasibility of the growth of large single crystals more than $16 \mathrm{~mm}$ in diameter by seeded growth. However, the quality of the crystals is still not sufficiently good for many applications. Recently, Mori et $a{ }^{16)}$ and Tsunesada et ${ }^{1{ }^{17}}{ }^{17}$ have developed a slope nucleation method (SNM) for DAST growth in which laserinduced nuclei could grow and eventually stand up for further growth on the grooves of a Teflon plate. With a very low growth rate $(0.1-0.2 \mathrm{~mm} /$ day at a cooling rate of about $0.1^{\circ} \mathrm{C}$ /day), DAST crystals with excellent quality and good electrooptical (EO) sampling performance were also obtained; the FWHM (full width of half maximum) of the rocking curve is 18.7 arcsec. Indeed, SNM could be a potential technique for the growth of high-quality DAST crystals. However, the number of nuclei is still difficult to control. Very often, too many crystals stick together making them useless in applications. Meanwhile, many small

${ }^{*}$ Corresponding author. E-mail address: cwlan@ntu.edu.tw crystals appear at the bottom of the vessel, thereby reducing the growth yield markedly. Therefore, the nucleation control with a considerably low nucleus formation is important in improving the growth method.

There are several methods of manipulating crystal nucleation behavior and further controlling crystal growth. Changing the growth temperature is an effective method of morphology control. At a low growth temperature, very thin crystals have been obtained using SNM by Sasaki's group in Japan. ${ }^{18)}$ The use of an effective solvent is another method. Rai et al. ${ }^{19)}$ also tested a methanol/ethanol mixed solvent, and observed that metastable zone width ${ }^{20}$ increases with increasing ethanol percentage in the solvent. The use of a chelating agent, such as EDTA, ${ }^{21)}$ could be useful as well in nucleation control. Indeed, several successful examples have been obtained for both organic and inorganic crystals. ${ }^{22-25)} \mathrm{A}$ chelating agent can suppress cluster formation by capturing metallic ions and thus increase metastable zone width. However, the concept of using a chelating agent on DAST growth, especially by SNM, has not yet been reported thus far. It is believed that the increase in metastable zone width could yield fewer, but larger, crystals with high quality. Therefore, in this study, the effect of EDTA the metastable zone width and the SNM growth of DAST crystals were investigated. The grown crystals were further examined for comparison. The experiments are described $\$ 2$. Section 3 presents the results and discussion, followed by conclusions in $\S 4$.

\section{Experimental}

DAST was synthesized by reacting 4-picoline and methyl toluenesulphonate at $1: 1$ molar ratio for $2 \mathrm{~h}$ and $30 \mathrm{~min}$, and treating the product (4-methyl- $N$-methyl pyridinium tosylate) with 4-N,N-dimethylaminobenzaldehyde in equivalent molar ratio for $6 \mathrm{~h}$ using piperidine as a catalyst. The synthesis process was carried out inside a glove box with nitrogen atmosphere and the synthesized DAST material was further purified by recrystallization using methanol as solvent below $50^{\circ} \mathrm{C}$. The twice-recrystallized materials from methanol were used for nucleation studies and SLM growth.

The nucleation experiments for measuring metastable zone width were carried out in small cells $(20 \mathrm{ml})$ with and without the addition of EDTA. As the synthesis and purification of DAST involved much time and effort, the 


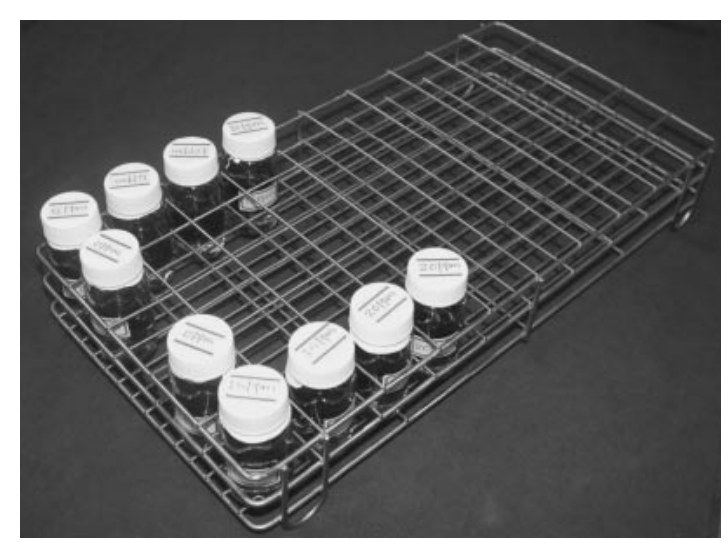

(a)

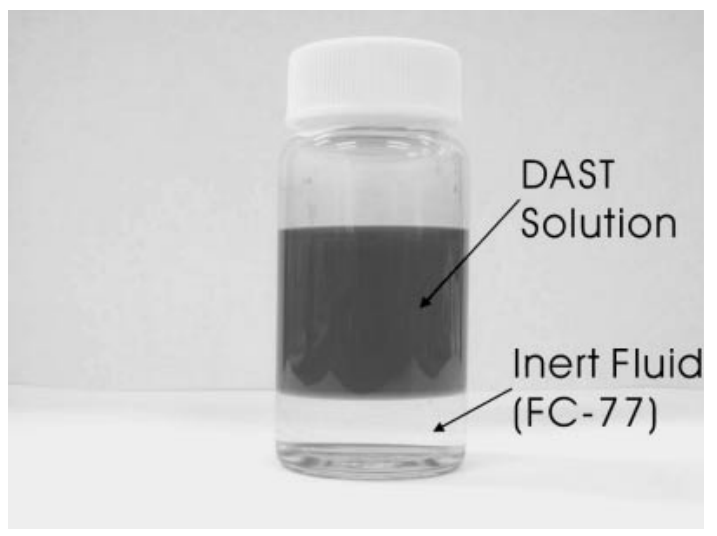

(b)

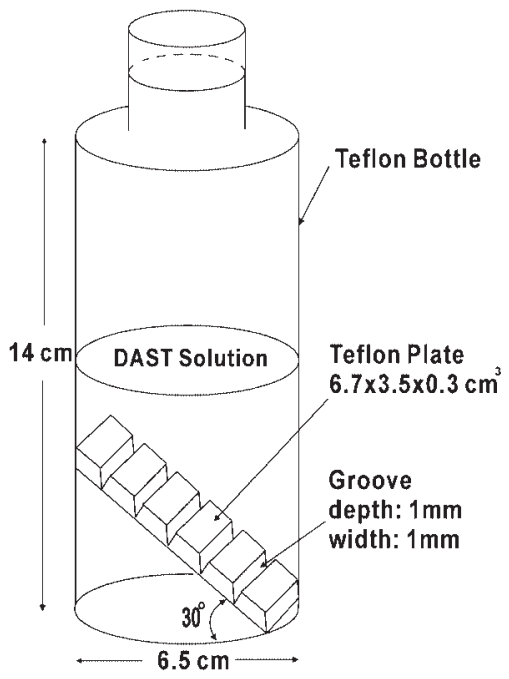

(c)

Fig. 1. (a) Schematic of small-cell (DAST solution) in nucleation experiment. (b) Close-up view of a small cell with DAST solution (top) and inert fluid (FC-77) (bottom). (c) Schematic of slope nucleation method.

"small-cell" experiments were extremely useful in performing several experiments with less amount of starting materials. Several experiments could be performed in a water bath for checking consistency and reproducibility. Also, the concentration gradient is less in a small cell. Therefore, the data could be more reliable. The small-cell experiments were carried out by putting growth cells into a tray, as shown in Fig. 1(a), and immersing the tray in a computer-controlled constant-temperature bath (CTB) for nucleation experiments. The CTB stability is within $\pm 0.01^{\circ} \mathrm{C}$. In each cell, to avoid the sticking of the crystals on the glass wall, an inert fluid, 3M Fluorinert electronic liquid FC-77, was used to let the DAST solution sit, as illustrated in Fig. 1(b); $5 \mathrm{ml}$ of preheated FC-77 inert fluid and $10 \mathrm{ml}$ of DAST solution were added for each cell. The clear interface between the inert fluid and the DAST solution was very helpful in the observation of nucleation. Furthermore, because FC-77 does not wet DAST crystals, it helped significantly in harvesting the DAST crystals grown while keeping the solvent from sticking to their surface.

To start the nucleation experiments, the solutions were kept at $50^{\circ} \mathrm{C}$ for 2 days for homogenization and then cooled to the respective saturation temperatures of $40^{\circ} \mathrm{C}, 35^{\circ} \mathrm{C}$, and $31^{\circ} \mathrm{C}$ at a cooling rate of $1^{\circ} \mathrm{C} / \mathrm{h}$. From the saturation temperature, the cooling rate was reduced to $1^{\circ} \mathrm{C} /$ day. Metastable zone width was measured by the conventional polythermal method. In this method, the equilibrium satu- rated solution was cooled from the overheated temperature until a visible crystal was observed. The observation was made in every $30 \mathrm{~min}$ for $3 \mathrm{~h}$ until the nucleation was found in the solution. After the growth, the DAST solution was pipetted out, and the crystals were immersed in the inner fluid for future experiments.

After having nucleation information from the small-cell experiments, effective growth conditions were adopted for the growth by SNM. The schematic of SNM is shown in Fig. $1(\mathrm{c})$. A given amount of EDTA $(0,10,20 \mathrm{ppm})$ was added to the solutions at the saturation temperatures of $35^{\circ} \mathrm{C}$ and $40^{\circ} \mathrm{C}$. The growth solutions were prepared as described in the earlier section. The DAST solution $(200 \mathrm{ml})$ was placed into two identical Teflon bottles at the saturation temperatures of $35^{\circ} \mathrm{C}$ and $40^{\circ} \mathrm{C}$ with and without EDTA, respectively. In each bottle, a clean and degreased Teflon plate $\left(6.7 \times 3.5 \times 0.3 \mathrm{~cm}^{3}\right)$ of $1 \mathrm{~mm}$ groove depth and $1 \mathrm{~mm}$ width was placed into the bottle having a slope of $30^{\circ}$ before the solution was poured, as shown in Fig. 1(c). Then, the bottles were closed tightly for experiments. Again, the growth solutions were maintained at $50^{\circ} \mathrm{C}$ for two days and cooled to the saturation temperature at a cooling rate at $1{ }^{\circ} \mathrm{C} /$ $\mathrm{h}$, and from the saturation temperature, the cooling rate was reduced to $1^{\circ} \mathrm{C} /$ day. The spontaneous nuclei generated in the solution condensed onto the slope and grew. A slower cooling rate, i.e., growth rate, could be used for realizing better-quality crystals; the effect of growth rate on crystal 
quality has already been investigated. ${ }^{26)}$ Therefore, we only chose one cooling rate for investigation here. After the growth, the crystals were harvested and stored in the FC-77 inner fluid for future measurements.

The crystals obtained from previous experiments were examined by several techniques. XRD (CAD4 Kappa Axis, Nonious, Netherlands) was used for checking the crystal structure and DSC (Model-2920, LT-Modulate, USA) for determining phase transition temperatures. However, no significant difference in what was observed by both methods between the crystals obtained with and without EDTA. Therefore, crystallinity was further examined by analyzing the XRD double-crystal rocking curve (Model RU-H3R, Rigaku, Japan) of the (001) surface of the SNM-grown crystals, where FWHM was measured for the best crystals (with the best surface morphology). Moreover, the eight best crystals selected from the experiments $\left(40^{\circ} \mathrm{C}\right.$ saturation temperature) were cold-mounted for Vickers hardness measurements using a micro-hardness tester (Model HMV2, Shimadzu, Japan) at a load of $490 \mathrm{dyne} / \mathrm{cm}^{2}$. Furthermore, the crystal absorbance from the visible to near-IR region was measured using a spectrometer (Jasco 570, Japan).

\section{Results and Discussion}

The amount of EDTA added was according to the solubility curve of EDTA in methanol. The solubility diagram of EDTA was drawn, and is shown in Fig. 2. As shown, EDTA has a very limited solubility in methanol. Too much of EDTA added caused the precipitation of EDTA crystals on DAST crystals leading to poor surface quality. On the other hand, if the amount of EDTA added was sufficient, no significant effect was found. It was found that $20 \mathrm{ppm}$ seemed to be the maximum amount of EDTA that could be added for the experiments (at $40^{\circ} \mathrm{C}$ ). At this amount, a small number of EDTA crystals were observed when the temperature was less than $31^{\circ} \mathrm{C}$. For a lower growth temperature, the amount of EDTA added should be reduced.

\subsection{Effect of EDTA on metastable zone width}

The metastable zone width of pure and EDTA-added DAST solutions were measured at different saturation

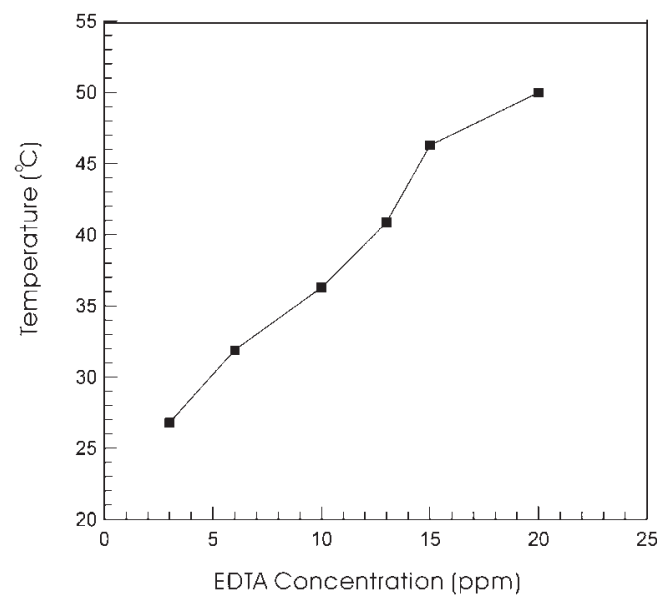

Fig. 2. Solubility curve for EDTA in methanol.

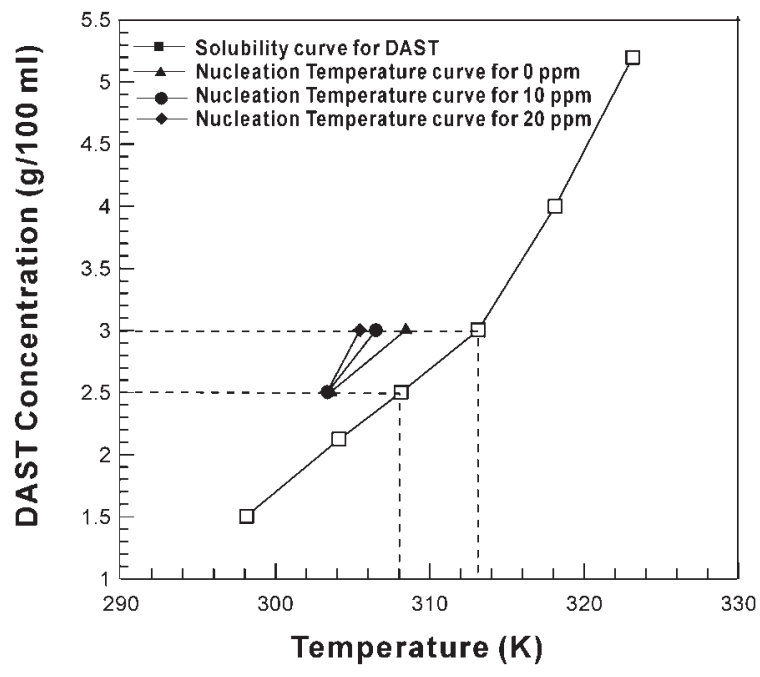

Fig. 3. Metastable zone width of EDTA-added DAST solution.

temperatures. The difference between the saturated temperature and the nucleation temperature was taken to be the metastable zone width of the system. It was found from the experiments that EDTA had no appreciable effect on suppressing the clusters in the solution prepared at a saturation temperature of $31^{\circ} \mathrm{C}$ due to the very low solubility of EDTA. Hence, the nucleation experiments with EDTA were carried out at the saturation temperatures of $35^{\circ} \mathrm{C}$ and $40^{\circ} \mathrm{C}$.

The nucleation temperatures for different EDTA concentrations at the saturation temperatures of $40^{\circ} \mathrm{C}$ and $35^{\circ} \mathrm{C}$ are shown in Fig. 3. The metastable zone widths of the DAST solutions prepared at a $40^{\circ} \mathrm{C}$ saturation temperature were about $4^{\circ} \mathrm{C}, 6^{\circ} \mathrm{C}$ and $7^{\circ} \mathrm{C}$ for the pure (0 ppm EDTA), $10 \mathrm{ppm}$, and $20 \mathrm{ppm}$ EDTA-added solutions, respectively. As shown, metastable zone width considerably increased with the addition of EDTA in solutions of higher saturation temperature $\left(40^{\circ} \mathrm{C}\right)$. However, it was not considerably affected by EDTA in the solution with a saturation temperature of $35^{\circ} \mathrm{C}$. This was believed to be due to the less dissociation of EDTA in solutions at lower temperatures caused by its restricted solubility.

\subsection{Crystal size distribution}

The harvested crystals in the nucleation experiments were classified according to their size, and the size distributions are given in Fig. 4. Because there were many tiny crystals for the nucleation without EDTA, weight was used rather than number in expressing size distributions. In order to check the consistency of the results of the experiments, five experiments were carried out and their results are compared in Fig. 4, where the crystal photographs from the first experimental run are shown for reference. As shown, the average crystal sizes were about 1-2 mm [Fig. 4(a)] and 2$3 \mathrm{~mm}$ [Fig. 4(b)] in pure and $10 \mathrm{ppm}$ EDTA-added DAST solutions at $35^{\circ} \mathrm{C}$, and about $1-3 \mathrm{~mm}$ [Fig. 4(c)] and 4-6 mm [Fig. 4(d)] in pure and 20 ppm EDTA-added DAST solutions at $40^{\circ} \mathrm{C}$, respectively. It is evidenced from the crystal size distribution that the crystals are smaller in the pure solution (0 ppm EDTA) at both lower and higher saturation temperatures. At the lower saturation temperature $\left(35^{\circ} \mathrm{C}\right)$, the 


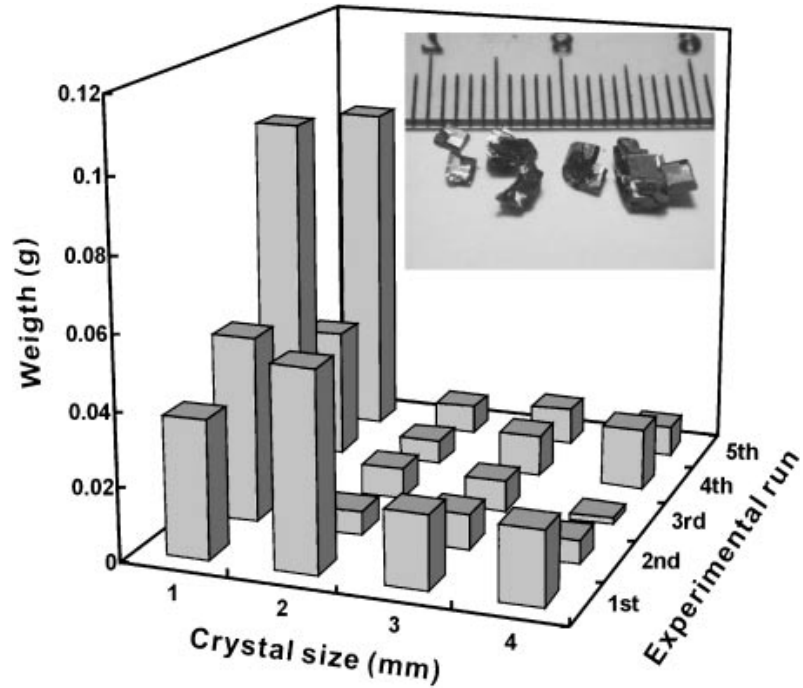

(a)

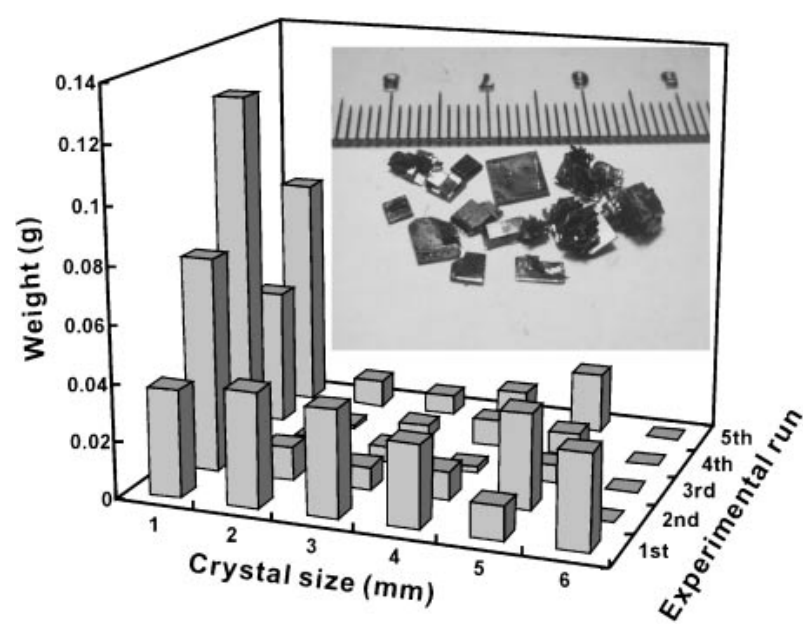

(b)

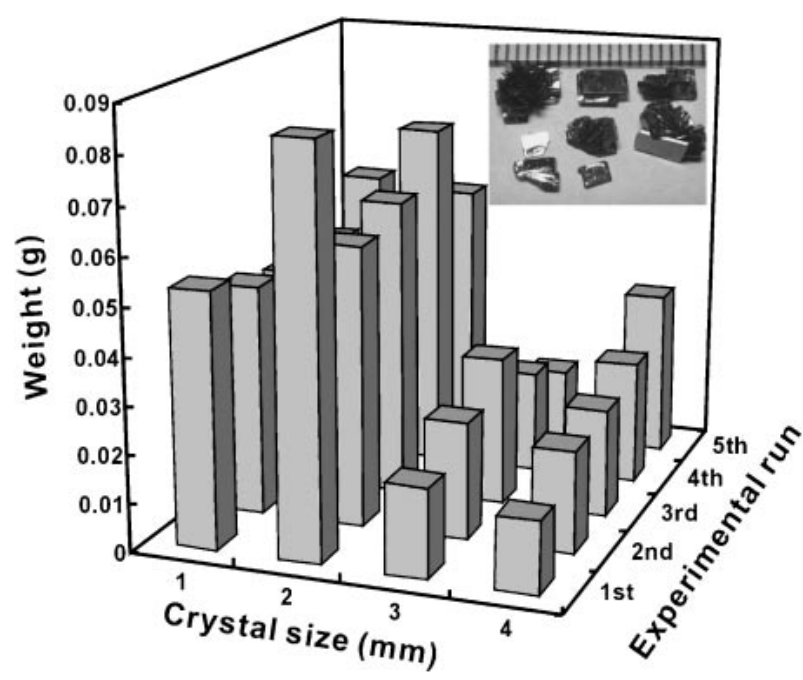

(c)

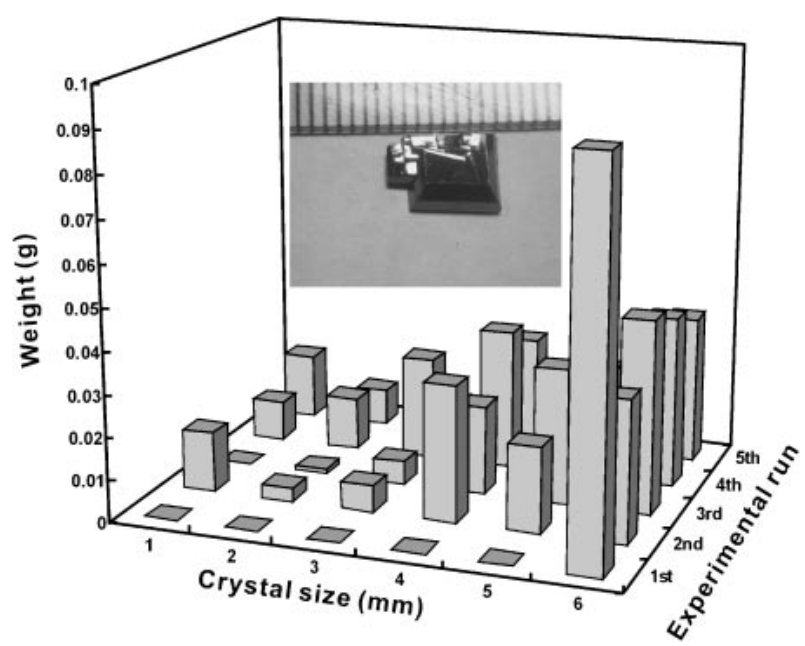

(d)

Fig. 4. (a) Crystal size distribution for "small-cell" experiment: pure DAST solution (0 ppm of EDTA) prepared at $35^{\circ} \mathrm{C}$ saturation temperature. (b) Crystal size distribution for "small-cell" experiment: $10 \mathrm{ppm}$ EDTA-added DAST solution prepared at $35^{\circ} \mathrm{C}$ saturation temperature. (c) Crystal size distribution for "small-cell" experiment: pure DAST solution (0 ppm of EDTA) prepared at $40^{\circ} \mathrm{C}$ saturation temperature. (d) Crystal size distribution for "small-cell" experiment: 20 ppm EDTA-added DAST solution prepared at $40^{\circ} \mathrm{C}$ saturation temperature.

crystals stuck together in the pure solution ( 0 ppm EDTA). However, with the addition of EDTA, the clustering phenomenon was markedly suppressed. Therefore, EDTA indeed improved crystal size, and decreased the number of crystals. The higher the saturation temperature $\left(40^{\circ} \mathrm{C}\right)$, the larger the crystal grown. The addition 20 ppm EDTA further reduced clustering to a great extent which in turn yielded bigger crystals. As shown in the first experiment whose results are shown in Fig. 4(d) (20 ppm EDTA-added solution), we only obtained one crystal. Furthermore, the crystals grown at the higher saturation temperature $\left(40^{\circ} \mathrm{C}\right)$ were found to be thicker, whereas the crystals grown at the lower temperature $\left(35^{\circ} \mathrm{C}\right)$ were thinner. This was also consistent with the observation by Sasaki's group ${ }^{18)}$ as well as with the Jackson $\alpha$-factor theory. ${ }^{27)}$ According to the Jackson's $\alpha$ factor theory, i.e., $\alpha=\Delta S / R \sim \Delta H_{\mathrm{sol}} / R T$, crystal growth at a lower temperature can have a large $\alpha$-factor, so that the crystals grown could have larger faces (more platelike). In the equation, $\Delta S$ is the entropy of the solution, $R$ the gas constant, and $\Delta H_{\text {sol }}$ the enthalpy of the solution, which is almost constant.

As shown Fig. 4, the results of different experimental runs were very consistent. The trends of the results were reproducible. On the other hand, adding too much EDTA, e.g., more than $25 \mathrm{ppm}$, yielded poor-quality crystals. The insoluble and precipitated EDTA particles stuck on the crystal surfaces. This is also the reason the allowable EDTA concentration at lower temperatures is low.

\subsection{Effect of EDTA on SNM growth}

The crystals grown using the slope nucleation method (SNM) with and without EDTA are shown in Figs. 5(a)5(d). Again, fewer, but larger, crystals were obtained from the EDTA-added solutions. Apparently, the number of 


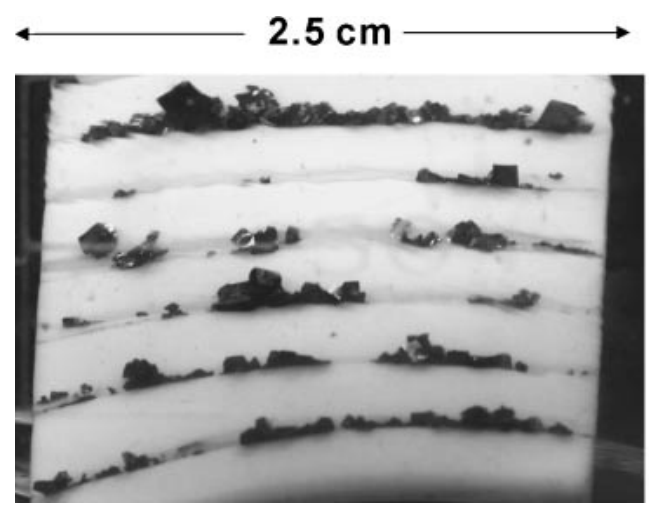

(a)

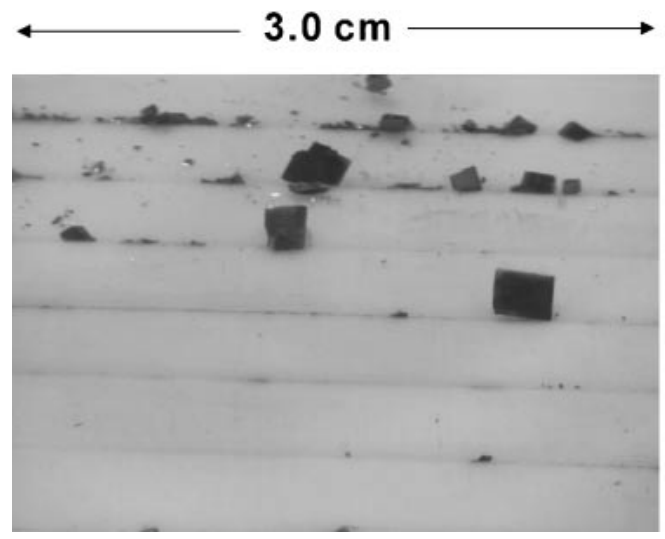

(b)

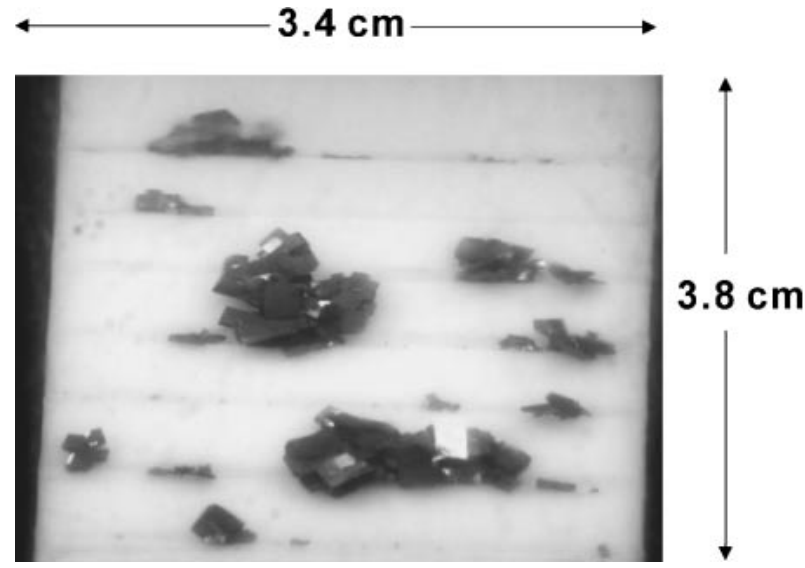

(c)

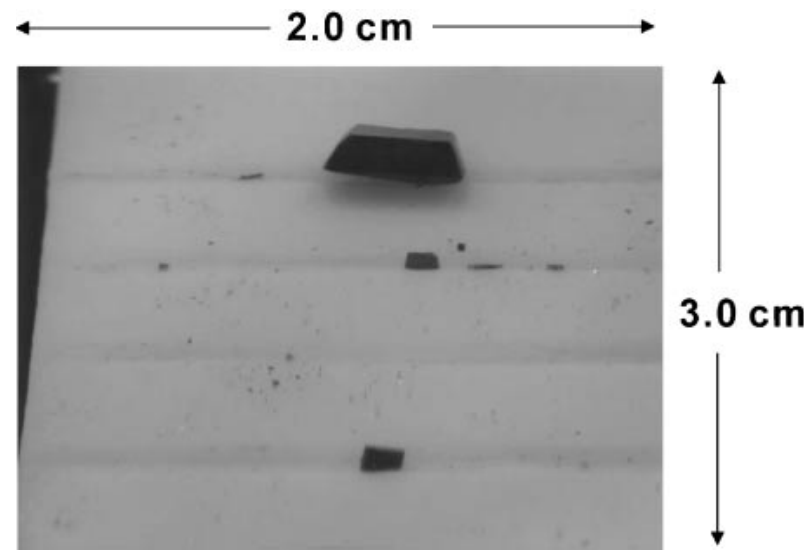

(d)

Fig. 5. (a) DAST crystals grown by slope nucleation method from the pure DAST solution prepared at $35^{\circ} \mathrm{C}$ saturation temperature. (b) DAST crystals grown by slope nucleation method from the $10 \mathrm{ppm}$ EDTA-added DAST solution prepared at $35^{\circ} \mathrm{C}$ saturation temperature. (c) DAST crystals grown by slope nucleation method from the pure DAST solution prepared at $40^{\circ} \mathrm{C}$ saturation temperature. (d) DAST crystals grown by slope nucleation method from the $20 \mathrm{ppm}$ EDTA-added DAST solution prepared at $40^{\circ} \mathrm{C}$ saturation temperature.

nucleation decreased in the EDTA-added DAST solutions. Moreover, the surface morphology of the crystals was considerably improved and the clustering of crystals was significantly minimized.

Because the cheators bind strongly to metallic ions if they can interact either $90^{\circ}$ or $180^{\circ}$ apart around the ions, the secondary nucleation due to the presence of impurities is suppressed. ${ }^{25)}$ From the metastable zone width diagram in Fig. 3, it was observed that the addition of EDTA increased the metastable zone width of the solutions (both at $35^{\circ} \mathrm{C}$ and $40^{\circ} \mathrm{C}$ saturation temperatures). Although the metastable zone width difference was not large between the pure and EDTAadded solutions at both saturation temperatures, especially at $35^{\circ} \mathrm{C}$ 's, from Fig. 5, the effect of EDTA seemed to be large in suppressing spurious nucleation and cluster formation. As shown, many nuclei were found to grow in the grooves of the slope and cluster together in pure DAST solution $(0 \mathrm{ppm}$ EDTA) at both the lower and higher saturation temperatures $\left(35^{\circ} \mathrm{C}\right.$ and $\left.40^{\circ} \mathrm{C}\right)$. In addition, the lower saturation temperature $\left(35^{\circ} \mathrm{C}\right)$ yielded smaller crystals than the higher saturation temperature $\left(40^{\circ} \mathrm{C}\right)$. However, with EDTA, as shown in Figs. 5(b) and 5(d), it can be concluded that the addition of EDTA to the growth solution at an optimum level increases the size and improves the morphology of the crystal. The clustering of nuclei was suppressed as well. It can be further observed from the results that the effect of chelators in suppressing clustering and spurious nucleation was more significant at $40^{\circ} \mathrm{C}$ than that at $35^{\circ} \mathrm{C}$. The trend of the crystal size distribution was the same for both small-cell and SNM experiments. Apparently, EDTA interacted strongly with DAST or impurity ions in the solution. This interaction might reduce the concentration of free DAST or metallic ions. As a result, the rate of crystallization was reduced. Thus, only a few crystals were obtained. This controlled crystallization became more probable with increasing EDTA concentration within the solubility limit. The enhancement of the metastable region by EDTA also increased the stability of the solution for nucleation.

\subsection{Characterizations}

The best crystals selected from the $40^{\circ} \mathrm{C}$ SLM growth were further examined by analyzing their double-crystal rocking curves. Figure 6 shows the XRD rocking curves for the (001) surface (the second largest facet of the crystal) of the DAST crystals grown from the solutions with and without EDTA. As shown, the crystallinity of the crystal 


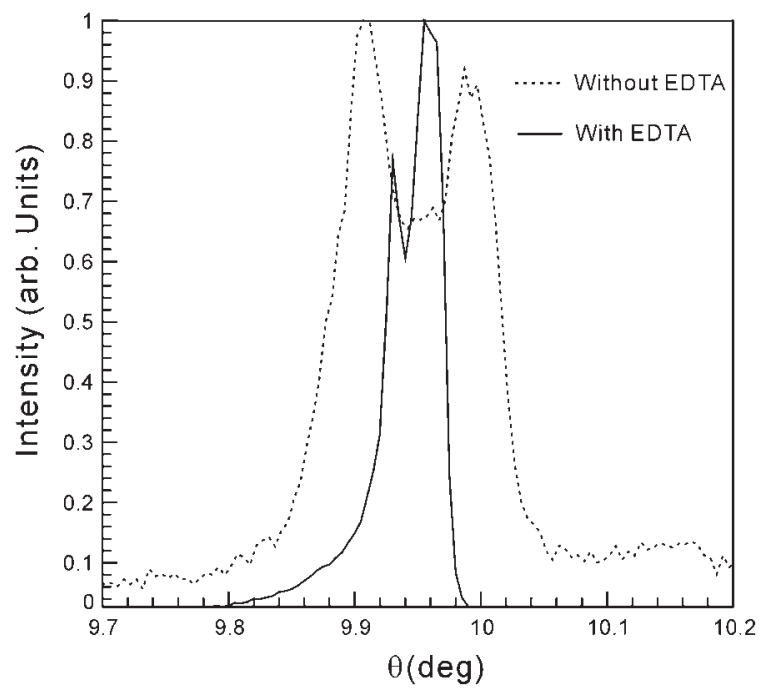

Fig. 6. XRD rocking curves for the DAST crystal growth by SNM with and without EDTA ( $20 \mathrm{ppm}$ at $40^{\circ} \mathrm{C}$ saturation temperature); cooling rate was at $1^{\circ} \mathrm{C} /$ day.

grown from the EDTA-added solution was much better than that grown in the solution without EDTA. The FWHMs of the two curves are about 170 and 475 arcsec, respectively. Again, because the impurities in the growth solutions were captured by chelators, crystals with higher quality were formed as expected. Although the FWHMs are larger than the highest FWHM reported by Sasaki's group, ${ }^{17,26)}$ they are still better than those reported based on the same cooling rate of $1{ }^{\circ} \mathrm{C} /$ day. Our result is comparable with that of the seeded growth at $0.18 \mathrm{~mm} /$ day in ref. 26 , which corresponds to a cooling rate of about $0.1^{\circ} \mathrm{C} /$ day. Furthermore, the Vickers hardness of the crystal obtained from the EDTA-added solution was significantly larger than those without EDTA. The average Vickers hardnesses of the four crystals (five indentations each) was about $50 \pm 7$ in the solution with EDTA and $30 \pm 8$ in the solution without EDTA. Clearly, the crystals grown in the EDTA-added solution were significantly harder and showed better mechanical properties. Furthermore, the hardness of the crystal grown in the solution with EDTA was slightly better than those reported by Adachi et al. ${ }^{26)}$ however, they did not report how much load was used for indentation. Therefore, further comparison was not possible. Nevertheless, it could be concluded that the crystals grown in the EDTA-added solution were significantly better in both crystallinity and hardness. These properties may be further improved if the growth rate is reduced as discussed in ref. 26. The optical absorbance in the visible/near-infrared region of the grown crystals was further examined using a Jasco 570 spectrometer, and a typical result is shown in Fig. 7. The crystal grown from the EDTA-added solution seemed to be more transparent. This could also be an indication of the better crystallinity of the crystals grown from the EDTA-added solution due to the slower nucleation process and the low cluster formation. In summary, based on the above consistent results with sufficient evidence, fewer, but bigger, crystals could be obtained using the chelating agent EDTA, which is a great advantage in crystal growth, and the crystals grown from the EDTA-added solution also had better quality, not just in

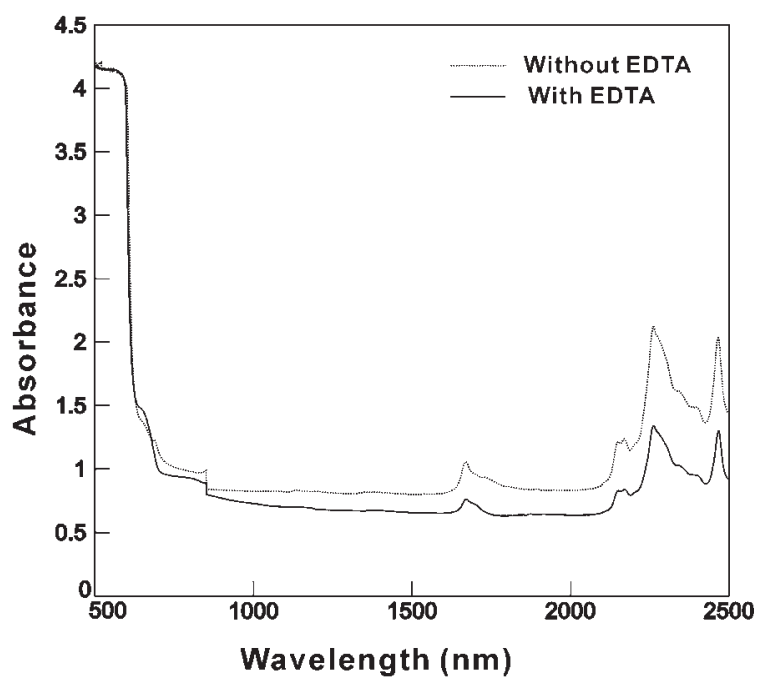

Fig. 7. Absorbance in the visible to near-IR region of the EDTA crystals grown by SNM with and without EDTA.

terms of surface morphology, but also in terms crystallinity, and mechanical and optical properties.

Moreover, the results obtained from the "small-cell" nucleation experiments will be utilized for seeded growth in the near future. A study using an optimum concentration of EDTA in the seeded growth is also in progress.

\section{Conclusion:}

Nucleation experiments (metastable zone width) were performed with and without the addition of a chelating agent at different saturation temperatures, which showed that the addition of the chelating agent EDTA increases metastable zone width. Also, they revealed that spurious nucleation and cluster formation in the solution could be suppressed significantly. Using the results obtained in metastable zone width experiments, the slope nucleation method growth of DAST was performed. Again, it was inferred that the addition of EDTA in the slope nucleation method could yield crystals with good morphology, and fewer crystals were grown in the grooves of the slope. The rocking curve measurements and hardness tests also indicated that the crystals grown in the EDTA-added solution have better crystallinity and mechanical properties. The transparency of the crystals in the visible/near-infrared region was also slightly improved. To grow crystals having sufficiently good quality for device applications, a much lower growth rate should be used; however, this is beyond the scope of this study.

\section{Acknowledgements}

CWL is grateful to Professor T. Sasaki and Professor Y. Mori, Department of Electrical Engineering, Osaka University, Japan, for their assistance in setting up this research project. The great hospitality and fruitful discussion provided by Professor Sasaki's group during CWL's visit in 1998 and 2002 are highly appreciated. We also acknowledge Professor Ito of Tohoku University for his valuable comments and suggestions. Valuable comments from the reviewer are appreciated. 
1) R. W. Munn and C. N. Ironside: Principles and Applications of Nonlinear Optical, Materials (Chapman \& Hall, London, UK, 1993).

2) P. Gunter: Non-linear Optical Effects and Materials (Springer-Verlag, Berlin, Germany, 2000).

3) N. Vijayan, R. Ramesh Babu, R. Gopalakrishnan, S. Dhanuskodi and P. Ramasamy: J. Cryst. Growth 233 (2001) 863.

4) R. Ramesh Babu, N. Vijayan, R. Gopalakrishnan and P. Ramasamy: J. Cryst. Growth 240 (2002) 545.

5) N. Vijayan, R. Ramesh Babu, M. Gunasekaran, R. Gopalakrishnan, R. Kumaresan, P. Ramasamy and C. W. Lan: J. Cryst. Growth 249 (2003) 309.

6) H. Nakanishi, H. Matsuda, S. Okada and M. Kato: Proc. MRS. Int. Mtg. Adv. Mater. 1 (1989) 97.

7) H. Nakanishi, H. Matsuda, S. Okada and M. Kato: Japan Patent Application No. Toku-Gan-Sho 61-192404, 1986.

8) S. R. Marder, J. W. Perry and W. P. Schaefer: Science 245 (1989) 626.

9) F. Pan, M. S. Wong, Ch. Bosshard and P. Gunter: Adv. Mater. 8 (1996) 592.

10) R. Spreiter, Ch. Bosshard, F. Pan and P. Gunter: Opt. Lett. 22 (1997) 564.

11) N. A. V. Dantzig, C. M. Planken and B. J. Huib: Opt. Lett. 23 (1998) 466.

12) T. J. Carrig, G. Rodriguer, T. S. Clement, A. J. Taylor and K. R. Stewart: Appl. Phys. Lett. 66 (1995) 121.

13) U. Meier, M. Bosch, Ch. Bosshard, F. Pan and P. Gunter: J. Appl. Phys. 83 (1998) 3486.

14) U. Meier, M. Bosch, Ch. Bosshard and P. Gunter: Synth. Met. 109
(2000) 19

15) S. Sohma, H. Takahashi, T. Taniuchi and H. Ito: Chem. Phys. 245 (1999) 359.

16) Y. Mori, Y. Takahashi, T. Iwai, M. Yoshimura, Y. K. Yap and T. Sasaki: Jpn. J. Appl. Phys. 39 (2000) L1006.

17) F. Tsunesada, T. Iwai, T. Watanabe, H. Adachi, M. Yoshimura, Y. Mori and T. Sasaki: J. Cryst. Growth 237 (2002) 2104.

18) T. Sasaki: Private communication

19) R. N. Rai, J. M. Jeng, C. Y. Tai and C. W. Lan: J. Chin. Inst. Chem. Engrs. 33 (2002) 461.

20) H. E. Buckley: Crystal Growth (John Wiley \& Sons, New York, 1951).

21) F. J. Welcher: The Analytical Use of EDTA (D.Van Nostrand Company, Canada, 1957).

22) K. Srinivasan, K. Meera and P. Ramasamy: J. Cryst. Growth 205 (1999) 457.

23) K. Srinivasan, K. Meera and P. Ramasamy: Cryst. Res. Technol. 3 (2000) 291.

24) N. P. Rajesh, K. Meera, K. Srinivasan, P. Santhana Raghavan and P. Ramasamy: J. Cryst. Growth 213 (2000) 389.

25) N. P. Rajesh, P. Santhana Raghavan, P. Ramasamy and C. W. Lan: J. Chin. Inst. Chem. Engrs. 33 (2002) 325.

26) H. Adachi, Y. Takahashi, J. Yabuzaki, Y. Mori and T. Sasaki: J. Cryst. Growth 198/199 (1999) 568.

27) K.A. Jackson: Materials Science Research (Plenum, New York, 1969) Vol. 4, p. 229. 